\section{Cuerpo y discurso psiquiátrico a comienzos del siglo XX en Uruguay: Insuficiencia, debilidad y adaptación}

\author{
Body and Psychiatric Discourse \\ in the Early Twentieth Century \\ in Uruguay: Insufficiency, \\ Weakness and Adaptation
}

\section{Corpo e discurso psiquiátrico nos primórdios do século XX em Uruguai: Insuficiência, debilidade e adaptação}

\section{Agustina Craviotto Corbellini*}

* Profesora de la Universidad de la República, Uruguay; estudiante de maestría en Estudios Interdisciplinarios de la Subjetividad, Facultad de Filosofía y Letras, UBA, Argentina. Investigadora del Grupo Políticas Educativas y Políticas de Investigación en Educación Física (GPEPI/ISEF/UdelaR) y del Grupo Formación de la clínica psicoanalítica en el Uruguay, Facultad de Psicología/ Udelar. Correo electrónico: agustinacraviotto@gmail.com

\section{Resumen}

Este estudio analiza el discurso médico psiquiátrico y la psicología experimental del siglo xx en el Uruguay, como saberes expertos sobre el niño y como referentes del discurso pedagógico de la época. Se presentan algunos elementos claves del desarrollo de la psiquiatría en el país y, a partir de ellos, se estudia cómo la disfuncionalidad del cuerpo se constituyó en el foco de análisis que habilitó una serie de clasificaciones de los escolares. Se toma como eje de reflexión la relación del cuerpo disfuncional con el aprendizaje escolar y se reconocen la indisciplina y las debilidades mentales como elementos que articulan los discursos psiquiátrico y psicológico con el discurso pedagógico, a través de la noción de adaptación.

\section{Palabras clave}

Cuerpo; discurso psiquiátrico; adaptación; funcionalidad

\section{Abstract}

This study analyzes the psychiatric medical discourse and experimental psychology during the twentieth century in Uruguay, as expert knowledge on children and as referents of the pedagogic discourse of that period. As an introduction some key elements of psychiatry in the country are presented; from them, it is stuyed how the dysfunction of the body became the focus of analysis that led to a series of school children's classifications. The relationship of the dysfunctional body with school learning is at the core of the reflection, and indiscipline and mental weaknesses are taken as elements articulating the psychiatric and psychological discourses with the pedagogical one, through the notion of adjustment disorder.

\section{Keywords}

Body; psychiatric discourse; adjustment disorder; functionality

\section{Resumo}

Este estudo analisa o discurso médico psiquiátrico e a psicologia experimental do século xx no Uruguai, em quanto saberes especializados sobre a criança e como referentes do discurso pedagógico dessa época. Apresentam-se alguns elementos chaves do desenvolvimento da Psiquiatria no país e, a partir deles, estudase como a disfuncionalidade do corpo constituiu-se no alvo da análise que habilitou uma série de classificações dos escolares. Toma-se como eixo de reflexão a relação do corpo disfuncional com a aprendizagem escolar e reconhece-se a indisciplina e as debilidades mentais como elementos que articulam o discurso psiquiátrico e o psicológico com o discurso pedagógico, através da noção de adaptação.

\section{Palavras-chave}

Corpo; discurso psiquiátrico; adaptação; funcionalidade

Fecha de recepción: febrero 26 de 2016 Fecha de aprobación: mayo 6 de 2016

................................................ 


\section{El discurso psiquiátrico en el Uruguay del $\mathbf{9 0 0}$}

$\mathrm{E}$ l avance de los estudios de la psique por parte de la medicina, a comienzos del siglo $\mathrm{xx}$, toma como objeto al niño; se produce una nueva narrativa y el desarrollo de tecnologías que se despliegan sobre estos cuerpos ahora reconocidos. Para el caso uruguayo, el 900 significó un momento de notables cambios en el campo de la medicina que alcanzaron gran auge la medicina infantil y la psiquiatría.

El establecimiento de los cuerpos de los niños como objeto de estudio permitió una universalización del saber psiquiátrico. Así pudo convertirse en cierta instancia de control de las conductas

no fue mediante la conquista de la totalidad de la vida ni con el recorrido del conjunto del desarrollo de los individuos desde su nacimiento hasta su muerte; al contrario, fue al limitarse cada vez más, al excavar cada vez más profundamente en la infancia. (Foucault, 2000, p. 285)

La tesis de Michel Foucault (2000) confirma que el estudio de los denominados retardados mentales es la vía por la cual la psiquiatría se apodera del terreno de la infancia y se inicia el proceso de psiquiatrización de la sociedad. La psiquiatría clásica le anuncia al mundo la llegada de otro: el enfermo mental; el discurso que le dirige al tiempo que lo crea como realidad instala

- un sufrimiento y una singularidad conocida por un experto: el médico,

- un conocimiento del sujeto dado por la mirada externa, no por el propio sujeto, pues "conocemos esa enfermedad lo bastante para saber que no puedes ejercer sobre ella y con respecto a ella ningún derecho" (Foucault, 2003, p. 394)

- sufrimiento que será llamado primero enfermedad y luego locura.

Un elemento fundamental sobre el saber psiquiátrico es la posición del médico como aquel que sabe, por lo tanto, es el responsable de la cura del paciente, como aquel que espera. Uno sabe, el otro espera; uno cura, el otro padece. Quien soporta el malestar es víctima de un cuerpo que no conoce. En el silencio del padecimiento se ubica la singularidad del sujeto y su malestar, su sufrimiento es el de cualquiera, por esto será tratado como cualquiera. No hay lugar para la palabra de quien sufre, más que para recabar datos hereditarios y señalar la forma del dolor; lo dicho se excluye de una singularidad, hay síntomas genéricos, nada tiene que decir que merezca ser escuchado por el saber de la ciencia médica.
Para el periodo definido en Uruguay, la psiquiatría define la normalidad partir de las virtudes y conductas burguesas (Barrán, 1991) y se produce una patologización y medicalización de la sociedad. El organicismo de origen positivista que imperaba en el campo médico construye la enfermedad mental en relación con causas orgánicas; desde este saber, la psiquiatría niega el sentido del discurso del loco y la histérica, calificado en la mayoría de los casos como mentiroso. La infección y el alcoholismo eran considerados antecedentes de la enfermedad mental, así también la sífilis, el mal uso de la energía en la sexualidad y la higiene, por lo que eran temas de preocupación y propaganda por parte de médicos y psiquiatras. La parálisis fue uno de los estados con mayor número de estudios.

La práctica psiquiátrica se inició sobre los casos médico-legales; se creó el primer "Asilo de dementes" en 1860, y en 1910 se inauguró el Manicomio Nacional Hospital Vilardebó. Los hospitales fueron dirigidos por médicos y progresivamente aquellos orientados hacia el estudio de la alienación mental de la psiquiatría clásica, con la influencia de la escuela médica francesa (Casarotti, 2007). En estas instituciones se crearon la Cátedra de Psiquiatría y en 1923 la Sociedad de Psiquiatría, que fueron los factores primordiales en el desarrollo de la psiquiatría nacional. Los médicos catedráticos de Psiquiatría completaron sus estudios en las principales clínicas europeas de la época.

La influencia de La Salpêtriére en el discurso médico del 900 en Uruguay es destacable; la escuela francesa de psiquiatría, y más específicamente aquella institución, significó uno de los más importantes aportes para la medicina uruguaya desde la segunda mitad del siglo XIX. Bernardo Etchepare ${ }^{1}$ viajó a Francia en 1910 y luego presentó a la Dirección de la Asistencia Pública Nacional el informe titulado Los débiles mentales (1912), también expuesto como Comunicación a la Sociedad de Medicina, en la sesión del 17 de julio de 1912. En el extenso informe, dio cuenta de la constitución de diversos "asilo-escuelas" del Viejo Continente, entre ellos La Salpêtriére; específicamente concentró su relato en la sección Félix Voisin, dirigida al tratamiento de las niñas débiles mentales.

En su informe, Etchepare destacó la importancia de haber visitado el hospicio "cuya fama ha llenado y llena aún el mundo entero científico" con los estudios de personalidades como Pinel, Morel, Trelat, Falret, Charcot y Marie, entre otros. En 1882, Jean Martin Charcot estableció la primera clínica neurológica

1 Bernardo Etchepare (1869-1925), es reconocido como el principal referente de la psiquiatría uruguaya. 
Clinique des Maladies du Système Nerveux, y tres años después Sigmund Freud permaneció cuatro meses en el hospital como su discípulo, con estudios sobre la histeria, la hipnosis y la sugestión. En esta revuelta epistémica se adjudicó el nacimiento del psicoanálisis; los estudios de Charcot llevaron a Freud a separar lo psicológico de las ataduras de lo anátomo-fisiológico, hasta su confirmación con el descubrimiento del inconsciente. Para el momento en que Etchepare realizó la visita a La Salpêtriére, Freud ya habría abandonado Francia y publicado la mayor parte de sus estudios, que dieron fuerza al discurso psicoanalítico. Sin embargo, Etchepare destacó como eminencias a J. P. Falret y a Félix Voisin, con sus "tratamiento médico-pedagógico de los débiles mentales" (1912, p. 246) de 1831 y la creación en 1834 de un establecimiento ortofrénico, respectivamente.

La relación directa de la psiquiatría uruguaya con La Salpetrière se dio a través Francisco Soca (18561922), quien realizó su tesis de doctorado llamada Etude clinique de la maladie de Friedreich, dedicada a su maestro Charcot, en 1888. Al regresar como miembro asociado extranjero de la Academia de Medicina de París encauzó sus estudios a la pediatría y se constituyó en el representante en Uruguay de la escuela francesa de medicina. Soca fue sucedido por Luis Morquio (1900), quien en 1900 fue nombrado profesor titular de Medicina Infantil y publicó el artículo "Displegia espasmódica familiar", texto fundamental por ser la primera mención al discurso psicoanalítico freudiano en el Uruguay (Craviotto et al., 2014).

\section{Cuerpos disfuncionales e insuficientes}

En un caso clínico (médico) de 1904 denominado Psicosis infantil (1905), Etchepare recuerda que en la infancia la psique del niño aún no se ha desarrollado y señala que

\section{la niñez, según Esquirol, está al abrigo de la locura. Según Anglade las verdaderas psicosis son raras en la infancia; lo que para este autor se presenta en ella es "la neurosis, la epilepsia, la corea, la histeria, el sonambulismo. (1905, p. 162)}

Las apreciaciones de este y otros casos clínicos (De León, 1903; Morquio, 1988, 1900, 1901; Etchepare 1904,1905 ) remiten a una psiquis infantil gobernada por el funcionamiento de la biología, y por lo tanto a sus trastornos como efecto de este mal funcionamiento. En 1905, Etchepare señala que

los estados mórbidos de la infancia [...] presentan una particularidad que los clasifica aparte en patología: es que los disturbios funcionales ó las altera- ciones de tejido que le corresponden, sorprenden órganos en vías de evolución, y en consecuencia la sintomatología está modificada según el grado más o menos elevado del desarrollo del órgano. (1905, p. 162)

Para Etchepare las enfermedades mencionadas como nerviosas, en su estructura y grado de gravedad están condicionadas a la edad del niño, por el tiempo de desarrollo de los órganos. Señala que "la primera infancia no puede señalar disturbios mentales de importancia, si es que realmente existen" (1905, p. 163) y pone seriamente en duda diagnósticos maniacos en niños recién nacidos, y niños de dos o tres años en Estados Unidos, ofrecidos como casos clínicos de la época. Esta etapa llamada "primera infancia" se entiende como una época vegetativa, donde no hay juicio ni ideas acabadas, donde el lenguaje recién empieza a aparecer defectuoso, lo que significaba la ausencia de intelectualidad. En esta línea, si no hay inteligencia organizada por el desarrollo completo de los órganos, no puede haber trastorno psíquico (Etchepare, 1905).

Etchepare (1905) cita en sus casos a Esquirol, quien fue representante del paradigma de la alienación mental junto con Pinel; sin embargo, menciona los estados mórbidos que lo ubicarían bajo un corrimiento teórico al paradigma iniciado por Bayle y Falret. Parado entre dos orillas, se refiere con Esquirol a un paradigma que delimitó categorías psicopatológicas estables, y pone como objeto de conocimiento el cuerpo orgánico, para afirmar la diferencia y aumentar la distancia con objetos espirituales, lógicos y éticos de la psicología y la filosofía. La posición científica buscada tuvo que dejar la observación de los comportamientos para no confundir el diagnóstico con temas subjetivos, definiendo la "enfermedad mental" como una disfunción orgánica estructural o producto de lesiones anatómicas o funcionales del encéfalo (Pompêo de Camargo, 2003). En segundo lugar, se refiere a un paradigma que sostuvo que la patología mental se componía de una serie de especies mórbidas y no como una enfermedad única. Este cambio sentó las bases para el paradigma de "las" enfermedades mentales, que puso el acento en la semiología, o estudio de los signos, y en la observación clínica del paciente por medio de la evaluación diagnóstica (Zlotnik, 2003). A pesar de esta diferencia, ambos paradigmas consideran la enfermedad mental o alienación, respectivamente, como una alternación orgánica.

Los casos de psicosis infantil fueron estudiados en niños de más de diez años, niños en época escolar. Se creía que las psicosis eran originadas por traumatis- 
mos o alguna infección y no habra diferencia alguna con una psicosis adulta (Etchepare, 1905). Etchepare describe el siguiente caso:

La historia que es objeto de esta comunicación es la de un estado maníaco, con alguna confusión mental, con los mismos síntomas que en el adulto [...] de un niño de 6 años, con herencia mental evidente, pero cuya psicosis no de sospechar fácilmente causa inmediata. [...] como en lo que á trastornos infantiles puede referirse en general, las causas morales determinante no existen. (1905, p. 164)

A partir de su formación neurológica, el psiquiatra uruguayo aclaró que ese caso, por lo extraño, era de utilidad para demostrar cómo el encéfalo sufre a esa edad (1905). Para su diagnóstico tomó principalmente los antecedentes hereditarios y el estudio somático respecto a la marcha, el habla, el control de esfínteres y el comportamiento con sus padres y el médico.

En 1912, Etchepare hizo una distinción fundamental diciendo que "no hay idiotez, hay idiotas; no hay debilidad mental, hay débiles mentales" (1912, p. 265). Si bien parece aclarar que cada individuo tiene sus particularidades, la condición intelectual entendida como razonamiento y posibilidad de adaptación define su condición de sujeto, ya no es algo que se posea, sino que se es. El diagnóstico cosifica al sujeto en su sentencia: "usted es débil" ${ }^{2}$ sentencia que lo ubica como incapaz de hacerse responsable de una locura que toma como objeto. No hay lugar para el sujeto en la debilidad, en la idiotez, en la locura, pues se ubica la enfermedad mental en el registro de un cuerpo fisiológico desprovisto de todo vestigio de alma, es decir, reduce la condición humana a la pura materia biológica.

La historicidad de cada sujeto queda subsumida en la información heredada y los efectos de la experiencia sobre lo orgánico, y la génesis de la debilidad como la interacción de aparatos que funcionarían según el "modo que permite expresar dicha interacción bajo la forma de una relación entre función y variable, que es lo que constituye su determinismo" (Lacan, 1966, p. 143).

\section{La alianza escuela-psiquiatría: indisciplina y debilidad mental}

En la escuela, los cuerpos de los niños son interrogados y demandados a partir del cuestionamiento de su participación en el aprendizaje. El escolar habita la institución educativa para incorporar aquellos conocimientos que le permitan acceder a la cultura y convivir con los otros. Ya en las primeras décadas del siglo $\mathrm{xx}$, la respuesta del escolar frente a lo ofrecido por la escuela habilitó lo que hoy, bajo otros nombres, es moneda corriente: la clasificación del niño avalada por la psiquiatría y la psicología experimental.

La palabra del médico fue la experta en temas escolares; la Escuela se constituyó como objeto de preocupación e intervención sobre "todos los problemas que se desarrollan [...] ora sea con el maestro, ora con el niño, ó ya con el medio donde estos se desenvuelven" (Rodríguez, 1910, p. 46). El principal asunto para atender por el saber psiquiátrico fue el identificar a los débiles apenas perceptibles (Etchepare, 1912). El conjunto de saberes de la psiquiatría y la psicología se ordenó por medio de la clasificación de los individuos, y en consecuencia se creó una extensa narrativa justificadora a partir de la pretendida comprobación científica de las capacidades y aptitudes.

En esta oportunidad revisamos dos nombres clasificatorios, por su importancia respecto del lugar del cuerpo y porque, a más de un siglo, permiten cuestionarnos su vigencia. Estos son:

El niño díscolo: Esta clasificación incluía aquellos que no incorporaban la disciplina y cuestionaban el poder adulto en la casa y en la escuela, es decir, no aceptaban la ley con la que el padre o maestro pretendían educarlo. El problema principal se situaba en los efectos de la indisciplina respecto al aprendizaje, puntualmente que no le permitiera alcanzar los distintos aprendizajes esperados en la escuela. El niño díscolo podía ser direccionado, reeducado, siempre bajo la amenaza de no poder civilizarse dada su incorregible naturaleza, y terminar en su fase adulta en los centros de corrección.

El niño con debilidad de aprendizaje: Con sus variantes y grados de dificultad, eran todos los niños posibles de ser nombrados con debilidad mental. La capacidad de trabajo era medible y posible de ser entrenada, su falta era una señal de anormalidad. Dado un conjunto de características, el nombrado enfermo era diagnosticado según la forma de debilidad mental, estructurada por una fórmula psíquica correspondiente a cada clasificación, que a su vez era correspondida con un tratamiento específico. (Etchepare, 1912)
2 Hoy podemos escuchar: "usted es depresivo", "usted es hiperactivo" y otra lista que avala el Manual diagnóstico y estadístico de los trastornos mentales (DSM por sus siglas en inglés). 
En cuanto a la primera clasificación, el carácter colectivo de la escuela ya estaba desde tiempo atrás bajo la lupa del médico higienista, quien determinaba la profilaxis necesaria para evitar los contagios de tuberculosis, difteria o escarlatina; sin embargo, ahora el médico higienista sabe que hay otras que atacan el intelecto. Rodríguez explica que

\begin{abstract}
la marcha normal de una escuela puede verse seriamente comprometida tanto por la presencia de un niño enfermo del cuerpo, como del intelecto [...] [al punto que] también hay que aislar ó dejar á un atrasado mental ó retardado, á un débil de espíritu ó á un anormal, porque tanto infectan y hacen peligrar al conjunto los primeros, como los segundos; aquellos que contagiando y transmitiendo á sus compañeros los síntomas de su enfermedad, y estos contagiando y transmitiendo su despreocupación, su indisciplina en el medio escolar que afecta el desarrollo intelectual de los niños normales. (1910, pp. 46-47)
\end{abstract}

El escolar indisciplinado debía corregirse, a riesgo no solo de su propia desgracia, sino de la de sus compañeros. La negación de atarse a la palabra del padre o la ley escolar podría esparcirse por las aulas como cualquier enfermedad viral.

Con relación a la segunda clasificación, el tratamiento de su gravedad fue auxiliado por los test provenientes de la psicología experimental, que cumplieron un papel fundamental en la identificación de estos casos debido a la medición objetiva de las capacidades intelectuales de los niños. La escala de normalidad de los estudios al igual que los diagnósticos psiquiátricos y psicológicos funcionaron como justificativos objetivos de la existencia de individuos inferiores y trastornados, frente a individuos normales y superiores. Los efectos del darwinismo llegaron de la mano de los discursos importados, a un país que lograba seguir los pasos del desarrollo de la ciencia en Europa, y que se apropiaba de una de las principales enseñanzas de este discurso evolucionista: la adaptación. Esta capacidad fue clave para la medicina y la escuela uruguaya. Binet ${ }^{3}$, quien un es referente para el país sobre estos temas, afirmaba que:

No hay nunca que perder de vista, cuando se habla de la educación, de la instrucción y de la formación de los espíritus, que toda actividad individual está sometida a una ley soberana: la adaptación del individuo a su medio; y que la enseñanza que se da a los jóvenes, teniendo por objeto aumentar el valor de tal adaptación, no debe ser juzgada más

3 Dedicado a la psicología experimental desde 1886, en 1892 comenzó a interesarse, con Simon, por la educación de los niños anormales. En 1907 Binet lanzó Las infancias anormales y había publicado Las ideas modernas acerca de los niños. que por la respuesta a esta pregunta capital: ¿la adaptación ha sido mejorada? He aquí nuestro criterio de pedagogía. (1911, p. 11)

El trabajo en la escuela se definió entonces por el grado de adaptación de los niños, lo que a su vez los clasificó según "los estudios de pedagogía fisiológica dignos del mayor apoyo por parte de todos los que se interesan por el porvenir de esta simpática agrupación" (Rodríguez, 1910, p. 47). La pedagogía queda subsumida a los datos suministrados por la fisiología, por la vía de la psiquiatría y la psicología experimental. Finalmente, la apuesta fue común: producir un pasaje del ser al deber ser, específicamente, conocer al niño para educarlo.

Las diferencias respecto a un tipo único de respuesta hicieron que la psiquiatría centrara su trabajo en fijar un orden de diferencias, clasificando a los individuos según sus conductas más o menos alejadas de los parámetros establecidos arbitrariamente y sustentados por el discurso de la racionalidad moderna. Se definieron las "debilidades mentales en general los diversos grados de retraso mental" (Etchepare, 1912, p. 265); el niño anormal, el débil, el retrasado debía conocerse para direccionarlo en la medida de lo posible hacia una normalidad a favor de la civilización. El porqué de estas diferencias, eje de la clasificación de los niños, fue la dimensión intelectual; los retrasos fueron explicados mediante la herencia y la degeneración. Etchepare (1912) afirma el concepto utilizado por Blin ${ }^{4}$, que explica que los polos estaban determinados por la incapacidad del niño de superar las dificultades de la vida. Esta clasificación a la luz de los test constituye una alianza entre la psiquiatría y la escuela; la primera se ocupa de fijar el dato y ubicarlos en su lugar, la segunda de desplegar los dispositivos necesarios para incorporar los objetivos civilizatorios.

En 1910, Sebastián B. Rodríguez, miembro del Cuerpo Médico Escolar Nacional, escribió un artículo llamado "La educación médico-pedagógica de los retardados" ${ }^{\text {, donde expresa que }}$

La higiene escolar tiene indudablemente que ir desarrollándose en orden progresivo y creciente, y con ella obteniéndose día á día nuevas conquistas, que vendrán á favorecer, no digo ya la parte correspondiente á la salud corporal del niño — verdad esta que ya ni se discute-, sino también lo que

4 Director de la Colonia de Vaucluse, lugar visitado durante su estadía en Europa el año previo al informe.

5 Trabajo presentado en agosto de 1909 en el IV Congreso Médico Latino-Americano celebrado en Río de Janeiro (Rodríguez, 1909). 
pudiéramos llamar la salud intelectual del mismo, llegando, por lo tanto, á producirse en el orden escolar un doble saneamiento, el del cuerpo y el de la mente, y garantizando un desarrollo sinérgico y científico, adaptable á las aptitudes físicas y á las capacidades psíquicas de cada escolar. (1910, p. 46)

El discurso psiquiátrico parece que no distingue cartesianamente entre una sustancia pensante y una sustancia extensa, que elimina la independencia entre sustancias; si los problemas de la psiquis son aquellos propios de una disfunción biológica, estas se reducen a un mismo fenómeno. Sin embargo, lo que implicó esta dicotomía mente/cuerpo fue dejar fuera el alma, que quedó ligada a la metafísica (Fernández, 2009). El "doble saneamiento" se refiere a la mente ligada a la razón, y al cuerpo como el lugar de la experiencia; ya no bastan los consejos dirigidos al cuidado del cuerpo, sino que también debe atender a la salud intelectual o de la mente. El rol del médico en la escuela se dirige a la civilización, el cuidado de la especie humana y por esto "se preocupa de la parte más noble e importante de nuestro ser, de la que depende nuestra gran supremacía sobre el resto de los seres vivientes que pueblan nuestro globo" (Rodríguez, 1910, p. 47). Tal nobleza y distinción le corresponde a la razón.

Las debilidades del cuerpo, sean tanto de orden psíquico como físico, son debilidades de los órganos. No se desconocen otros factores adyacentes, principalmente los vinculados a la moral, estos también se ubican en los diagnósticos, pues son otros elementos que conforman el cuadro y por lo tanto son dignos de tratamiento y cura. El cuerpo revelado en la disfunción es aquel que el saber médico conoce, y ese supuesto saber es el que le dice que puede curarlo. Higiene, prevención, test, tratamientos correctivos morales y farmacológicos permiten al experto restablecer la "salud mental"; se trata de volver al camino al niño desviado, de traerlo de vuelta a su natural funcionalidad.

El camino "normal" de la escuela del 900 luchaba contra un fenómeno de trastorno mental infantil pensado como un retraso, que se llamaba idiocia (Etchepare, 1912) y estado patológico de inadaptación. Los significados incorporados por la modernidad sobre el significante niño encuentran un lugar en una historia paralela y solidaria a la mutación en la concepción del tiempo en Occidente; como efecto sobre los cuerpos en la constitución de la historia, se reduce a una cuestión cronológica, a un "antes-después". Agamben señala que "la representación del tiempo como homogéneo, rectilíneo y vacío surge de la experiencia del trabajo industrial y es sancionada por la mecánica moderna que establece la primacía del movimiento rectilíneo uniforme con respecto al circular" (2004, p. 140). Como parte de ese relato el niño se introduce en esa dinámica del antes-después, en ese proceso evolutivo que tiene a dejar atrás algo para dirigirse a un nuevo estado posterior y natural. El infans no será aquel que puja en la entrada al mundo de lo simbólico, escindido entre la lengua y el habla, sino como positividad constatable de una fase o etapa cronológica por superar. En este proceso, la psiquiatría y la psicología ofrecen los conocimientos necesarios para el direccionamiento de las etapas en su aparente normalidad; la clasificación de la psiquiatría y los aportes derivados de los test de la psicología experimental fueron fundamentales a la hora de analizar cómo fue pensado el aprendizaje en la escuela.

El concepto clave de adaptación de estos discursos de afectación darwiniana pone la problemática del aprendizaje como algo temporal. El problema del aprendizaje fue fundamental en los llamados débiles sutiles (Etchepare, 1912), aquellos que no adquirían lo esperado para determinado momento mostraban un retraso y, en consecuencia, una debilidad. El control de los aprendizajes debía extenderse desde el comienzo de la etapa escolar con la finalidad de detectar los posibles débiles escondidos, pues estos eran un verdadero peligro ya que afectaban la velocidad y el ritmo de los demás, y alteraban el orden planificado.

Etchepare estableció una etiología de los débiles mentales dirigida a los tratamientos médico-pedagógicos; disponer de información como el origen, la forma y la intensidad de la inteligencia permitió conocer las posibilidades, y advertir a los educadores (maestros y médicos) para que no incurrieran en "optimismos exagerados ó en desencantos inoportunos é inmotivados" (Etchepare, 1912, p. 265). Los conocimientos eran comprobados y los caminos trazados, para una tarea que consistía únicamente en conocer este proceso que implica un continuo progresivo de etapas por superar. Etchepare señaló que

El término "retardado" ó "atrasado" indica más bien ó por definición un simple retardo ó atraso en la marcha de la inteligencia, suponiendo dejando suponer más bien, la idea de una normalidad posible, susceptible de ser alcanzada siempre, lo que no es cierto en la inmensa mayoría de los casos. (Etchepare, 1912, pp. 265-266)

Estos eran principalmente aquellos deficientes en el camino del desarrollo evolutivo de los órganos del aparato psíquico. El aprendizaje se entendía entonces como las adquisiciones medibles, enmarcadas en un tiempo que es el tiempo de producción normal. El niño débil era signo de la improductividad; estos principalmente debían dirigir sus esfuerzos a una 
homogeneización a partir de una moral acomodada a la demanda social, de una época con la que el niño debía identificarse para ser normal.

En pleno auge de las psicologías auxiliadoras de las pedagogías, se produjo un quiebre teórico con los aportes freudianos, el sujeto del inconsciente implicó una fuerte revisión de conceptos centrales que sostenían; para este caso, que el sujeto no es su inteligencia, ni es un organismo que se adapta. Los aportes del psicoanálisis fueron fundamentales para poner en discusión lo hasta entonces indiscutido de las psicologías evolutivas: no hay tal desarrollo ideal, progresividad, etapas preestablecidas (donde hay una unidad psíquica, un yo, una unidad). El psicoanálisis propone un sujeto que no puede ser sin el lenguaje; este como acontecimiento permite, en la formulación del discurso, que el aprendizaje entre en juego. El mismo Freud escribió que el inconsciente no conoce el tiempo por cuanto su repetición y su insistencia en cierto sentido convierte "al deseo en indestructible", lo inconsciente es algo que "insiste", que viene de las profundidades del pasado, que en cierto sentido nada puede satisfacer ni modificar: es un elemento completamente paradojal, que parece ir contra toda referencia biológica. La insistencia en la repetición inconsciente es una cosa que es preciso reelaborar en las categorías del tiempo, que es distinto del simple fluido temporal (Lacan, citado en Carusso, 1969).

Sintetizando, el discurso de comienzos de siglo xx sobre el escolar es el del control de las adquisiciones o del aprendizaje en su significado psicológico. Esto implicó la noción de desarrollo psicológico expuesta, en su dimensión lineal como proceso natural anclado en la estabilidad psíquica de un sujeto concebido como una unidad bio-psico-social. El significante niño puesto en representación como individuo capaz de sostener una relación transparente, clara y "sana" entre el saber y el lenguaje, a partir del equilibrio mudo del organismo.

\section{A modo de cierre}

El desarrollo teórico anterior presentó algunas referencias del discurso médico, específicamente el psiquiátrico, sobre cómo colocó al escolar como centro de las preocupaciones, como objeto de conocimiento. La constitución de los discursos sobre el escolar en Uruguay, a principios del siglo $\mathrm{xx}$, encontró asidero entre los desarrollos del discurso psiquiátrico de Esquirol y Falret, y la psicología experimental de Binet; y distó de los postulados psicoanalíticos iniciados por Freud en la misma época.
Tales discursos fueron dirigidos a la escuela, donde los cuerpos fueron interrogados y demandados a partir del cuestionamiento de su participación en el aprendizaje. El discurso bio-médico fue el principal exponente sobre la especificidad psíquica y somática infantil, y la pedagogía su principal foco de acción, lo que dio lugar a estudios y tratamientos médico-pedagógicos. Estos, que se constituyeron como saberes expertos sobre el niño en su tiempo escolar, como etapa evolutiva en desarrollo, lo nombraron como inferior, incompleto y débil. Lo clasificaron según el parámetro adulto de la razón y se lo depositaron en la única empresa dirigida al tratamiento de sus debilidades y al fortalecimiento de sus aptitudes normales de adaptación.

El escolar fue interpelado a partir de las posibilidades de su inteligencia, según una fórmula psíquica, y la historicidad de cada sujeto quedó subsumida en la información heredada y en los efectos de la experiencia sobre lo orgánico. El discurso psiquiátrico, con la apoyatura de la psicología, remitió a una psiquis infantil gobernada por el funcionamiento de la biología, y por lo tanto a sus trastornos como efecto de este mal funcionamiento. Las enfermedades nerviosas, en su diversidad de estructura y grado de gravedad, estaban condicionadas a la edad del niño como tiempo medido por el desarrollo de los órganos. Si los problemas de la psiquis fueron aquellos propios de una disfunción biológica, estas se redujeron a un mismo fenómeno: el cuerpo orgánico.

Respecto al aprendizaje fueron dos las grandes clasificaciones (Etchepare, 2012): el niño díscolo, que era aquel reconocido como indisciplinado o que no respetaba la ley, y el niño con debilidad de aprendizaje, que se subdividía según las características de su debilidad mental.

Frente a este discurso tomado por la biología, el psicoanálisis lanzó un saber a partir de los aportes de Freud, quien rompió con la noción de sujeto para pensar al niño de otro modo, que apenas llegaron a escucharse en el Uruguay del 900 . Habrá que esperar varias décadas para que Freud adquiera otro estatus y deje (en parte) de ser leído en su perspectiva organicista. Los principales referentes del discurso psiquiátrico para la infancia, como Etchepare y Morquio, fueron quienes hicieron las primeras referencias al Freud neurólogo.

La indagación presentada abre varias preguntas sobre la vigencia de la relación cuerpo-aprendizaje en la escuela. ¿Qué continuidades encontramos entre estos discursos y los de la actualidad? ¿Qué distancia hay entre aquel imaginario de la conducta como una enfermedad biológica y la actual propuesta del cognitivismo? ¿Qué distancias hay entre el niño 
indisciplinado y débil mental, con el hiperactivo y con las variantes clasificatorias de las "dificultades de aprendizaje"? ¿Qué tan disparatadas o aberrantes fueron las clasificaciones y las prácticas correctivas morales y farmacológicas dispuestas en el 900, sobre las clasificaciones y prácticas sostenidas hoy por la industria farmacéutica y las neurociencias?

\section{Referencias}

Agamben, G. ([1978], 2004). Infancia e historia. Ensayo sobre la destrucción de la experiencia (2. ㄹ ed.). Buenos Aires: Adriana Hidalgo Editora.

Ardao, A. (1951). Batlle y Ordóñez y el positivismo filosófico. Montevideo: Número.

Barrán, J. ([1990] 1991). Historia de la sensibilidad en el Uruguay (tomo 2). El disciplinamiento (1860-1920). Montevideo: Ediciones de la Banda Oriental.

Behares, L. (2004). Enseñanza-aprendizaje revisitados. Un análisis de la "fantasía" didáctica. En L. E. Behares (dir.) Didáctica mínima. Los acontecimientos del saber (pp. 11-30). Montevideo: Psicolibros-Waslala.

Binet, A. ([1911] 2003). Las ideas modernas acerca de los niños. Biblioteca Virtual Universal, Editorial del Cardo. Recuperado de http://www.biblioteca.org.ar/ libros/89125.pdf

Carusso, P. (1969?). Pablo Carusso entrevista a Jaques Lacan. Recuperado de http://www.antroposmoderno. com/antro-articulo.php?id_articulo=812

Casarotti, H. (2007). Breve síntesis de la evolución de la Psiquiatría en el Uruguay. Montevideo: Revista de Psiquiatría del Uruguay, 71 (2), 153-163.

Craviotto, A.; García, F.; Moraes, M. y Mortola, V. (En proceso de edición). Recepción de las ideas freudianas en el Uruguay: cronología y datos para un estudio comparativo. Ponencia presentada en la mesa Capes-Udelar, en la XIX Jornada Corpolinguagem / VI Encontro Outrarte - Práctica da letra, uso do inconsciente - realizado en noviembre del 2014, en el Instituto de Estudos da Leinguagem da Universidade Estadual de Campinas.

De Lajonquier, L. (2011). Figuras de lo infantil. Buenos Aires: Nueva Visión.

Fernández, A. (2006). "Lo niño” y el psicoanálisis: ¿posibilidad o imposibilidad? [número especial]. Educacao Tematica Digital, (8) 20-48.

Fernández, A. (2014). El aprendizaje en cuestión, Montevideo: Editorial De la Fuga, FHCE, Udelar.
Fernández, A. (En proceso de impresión). Hablar de cuerpos. Saber de (los) cuerpos. En: Actas del XII Encuentro Nacional VII Internacional de Investigadores en Educación Física. De próxima publicación en: www.isef.edu.uy.

Foucault, M. ([1973-1974] 2000). El poder psiquiátrico. Buenos Aires: Fondo de Cultura Económica.

Lacan, J. ([1936] 2013). Más allá del Principio de realidad. En Escritos 1. Buenos Aires: Siglo xx editores.

Lacan, J. ([1966] 2002). Acerca de la causalidad psíquica. (T. Segovia, trad.). Escritos 1 (pp. 142-183). Buenos Aires: Siglo XXI editores.

Lacan, J. (1954-1955). El Seminario. Libro II. El yo en la teoría de Freud y en la técnica psicoanalítica. Buenos Aires: Paidós. Versión digital Psicolibros.

Méndez, E. (1975). Historia uruguaya (tomo 5). El Uruguay de la modernización. 1876-1904. Montevideo. Ediciones de la Banda Oriental.

Pompêo, M. (2003, junio). Esquirol e o surgimento da psiquiatría contemporânea. Revista Latinoamericana de Psicopatología Fundamental, 6 (2), 152-157. Recuperado de http://www.redalyc.org/articulo. oa?id=233018066011

Vomero, F. (2014). La psiquiatrización de la infancia en el Uruguay. Parte I: Bernardo Etchepare y el nacimiento de los débiles mentales. Recuperado de http://articulando.com.uy/2014/10/13/ la-psiquiatrizacion-de-la-infancia-parte-i/

Zlotnik, M. (2003). La psiquiatría clásica y sus dos clínicas. R. Mazzuca, C. Codoy, F. Schejtman y M. Zlotnik, Psicoanálisis y psiquiatría: encuentros y desencuentros. Buenos Aires: Bergasse.

\section{Fuentes consultadas}

Etchepare, B. (1905). Displegia espasmódica familiar. Revista Médica del Uruguay, 11 (3), 1900.

Etchepare, B. (1905). Psicosis infantil. Revista Médica del Uruguay, 162-168.

Etchepare, B. (1912). Los débiles mentales. Revista Médica del Uruguay, 16 (16).

Morquio, L. (1899). Parálisis dolorosa de los niños. Revista Médica del Uruguay, 10 (11).

Rodríguez, S. (1910). Educación médico-pedagógica de los retardados. Revista Médica del Uruguay, 46-54.

Ventura, C. y Puppo, H. (s. f.). Santin Carlos Rossi (18881936). En H. Gutiérrez Blanco. Médicos ejemplares uruguayos. Recuperado de http://www.smu.org.uy/ publicaciones/libros/ejemplares_ii/art_38_rossi.pdf 